\title{
Composite neuroendocrine carcinoma and squamous cell carcinoma with regional lymph node metastasis: a case report
}

\author{
Shintaro Fujihara ${ }^{1 *}$, Masahiko Kobayashi ${ }^{2}$, Masako Nishi ${ }^{1}$, Tatsuo Yachida ${ }^{3}$, Akira Yoshitake ${ }^{1}$, Akihiro Deguchi $^{1}$,
} Atsushi Muraoka ${ }^{2}$, Hideki Kobara ${ }^{3}$ and Tsutomu Masaki ${ }^{3}$

\begin{abstract}
Background: Neuroendocrine cell carcinoma is a rare variant of esophageal carcinoma. The characteristic clinical features and diagnosis of superficial neuroendocrine cell carcinoma remain to be established. We report a rare case of superficial coexistence of neuroendocrine cell carcinoma with squamous cell carcinoma treated by endoscopic submucosal dissection, and regional lymph node metastasis was detected after additional surgical treatment.

Case presentation: A 77-year-old Japanese man with esophageal squamous cell carcinoma received endoscopic submucosal dissection in en-bloc resection. Histopathological findings showed that lymphovascular invasion by the neuroendocrine cell carcinoma component occurred in the deep part of the muscularis mucosa. Regional lymph node metastasis was identified after additional surgical treatment. After surgical treatment, our patient received chemotherapy consisting of etoposide and carboplatin for 3 months. He is alive and shows no sign of disease recurrence 12 months after surgery.

Conclusions: This case report highlights the fact that even if neuroendocrine cell carcinoma is small and limited to superficial, the tumor has the potential for metastasis if lymphovascular invasion by the neuroendocrine cell carcinoma component occurs. In addition, this case indicates the necessity of close follow-up of small neuroendocrine cell carcinoma after treatment.
\end{abstract}

Keywords: Neuroendocrine cell carcinoma, Esophagus, Squamous cell carcinoma, Endoscopic submucosal dissection, Metastasis

\section{Background}

Neuroendocrine cell carcinoma (NEC) is rare variant of esophageal carcinoma. NEC is less common than squamous cell carcinoma (SCC) and adenocarcinoma, and it is a relatively rare disease with a reported incidence between $0.4 \%$ and $2 \%$ [1-3]. The prognosis of NEC of the esophagus is poor because the tumor is often at an advanced stage of disease at diagnosis. Superficial NEC of the esophagus is rare. To the best of our knowledge, only a few superficial NECs of the esophagus have been reported in the English literature [4]. Accordingly, the characteristic

\footnotetext{
* Correspondence: joshin@med.kagawa-u.ac.jp

'Department of Gastroenterology, Kagawa Rosai Hospital, 3-3-1, Joutou,

Marugame, Kagawa, Japan

Full list of author information is available at the end of the article
}

endoscopic features and diagnosis of superficial NEC remain to be established.

Endoscopic mucosal resection (EMR) and endoscopic submucosal dissection (ESD) offer non-invasive treatment for esophageal cancer limited to the mucosa and without lymph node metastasis [5]. ESD has a significantly higher curative resection rate and lower local recurrence rate than EMR, particularly in lesions less than $2 \mathrm{~cm}$ [6]. However, whether endoscopic treatment is sufficient for disease control for superficial NEC is unclear.

We report a rare case of superficial coexistence of NEC with SCC treated by ESD, and regional lymph node metastasis was detected after additional surgical treatment.

(c) The Author(s). 2018 Open Access This article is distributed under the terms of the Creative Commons Attribution 4.0 International License (http://creativecommons.org/licenses/by/4.0/), which permits unrestricted use, distribution, and reproduction in any medium, provided you give appropriate credit to the original author(s) and the source, provide a link to the Creative Commons license, and indicate if changes were made. The Creative Commons Public Domain Dedication waiver (http://creativecommons.org/publicdomain/zero/1.0/) applies to the data made available in this article, unless otherwise stated. 


\section{Case presentation}

A 77-year-old Japanese man presented to our hospital with esophageal mucosal abnormality in the middle thoracic esophagus. This abnormality was discovered in a barium study for a health checkup. His medical history was significant for primary hypertension. He reported a 50-year history of smoking 8-10 cigarettes per day. There was no family history. Physical and neurological examinations were unremarkable. Esophagogastroduodenoscopy showed 20-mm, reddish, elevated, and flat lesions in the middle thoracic esophagus (Fig. 1a). Narrow band imaging (NBI) endoscopy showed dot-like microvessels in elevated lesions (Fig. 1b, c). However, the microvascular pattern showed irregular, fine, reticular blood vessels, of Japan Esophageal Society (JES) classification type R, near the center of the lesions (Fig. 1d). Magnifying endoscopy with NBI revealed type B1 in elevated area, and type $R$ near the center of the lesion in the JES classification. Endoscopic ultrasound showed that the lesion was localized in the mucosa (Fig. 1e).

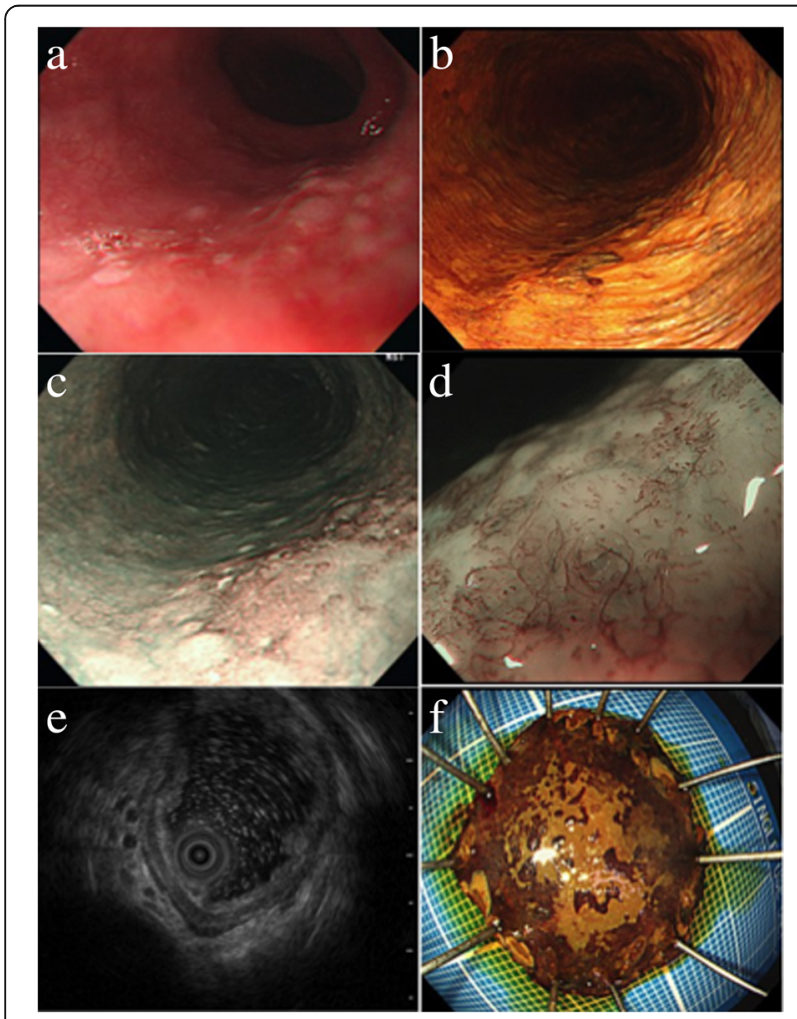

Fig. 1 a Esophagogastroduodenoscopy shows an irregular, reddish, flat lesion in the posterior wall of the mid-esophagus. b Lugol's iodine chromoendoscopy shows an unstained lesion that is located in the posterior wall of the mid-esophagus. c Narrow band imaging endoscopy shows a brownish area. d Magnifying endoscopy with narrow band imaging shows a microvascular pattern with irregular, fine, reticular blood vessels near the center of the lesion. e Endoscopic ultrasound image of the lesion limited to the mucosa. $\mathbf{f}$ En bloc resection by endoscopic submucosal dissection
Therefore, this area of invasion was: cancer limited to the epithelium (EP)/cancer invading into the lamina propria (LPM) to cancer invading into the muscularis mucosa (MM). Biopsies showed SCC of the esophagus. Computed tomography (CT) showed no evidence of lymph node and distant metastases. En bloc resection of the tumor was performed successfully by esophageal ESD without any complications (Fig. 1f).

Histopathological findings showed an admixture of endocrine cell tumor with SCC with an invasion depth into the muscularis mucosa (Fig. 2a). No complications were related to the procedure. Immunohistochemical analysis showed positivity for CD56, chromogranin, and synaptophysin in the NEC component (Fig. 2b-f). Small cell type NEC was arranged in a sheet fashion existing in the center of the tumor, and these were partially surrounded by well-differentiated SCC. Lymphovascular invasion of the NEC component occurred in the deep part of the muscularis mucosa. Our patient underwent additional surgical treatment consisting of video-assisted thoracoscopic esophagectomy, three-field lymph node

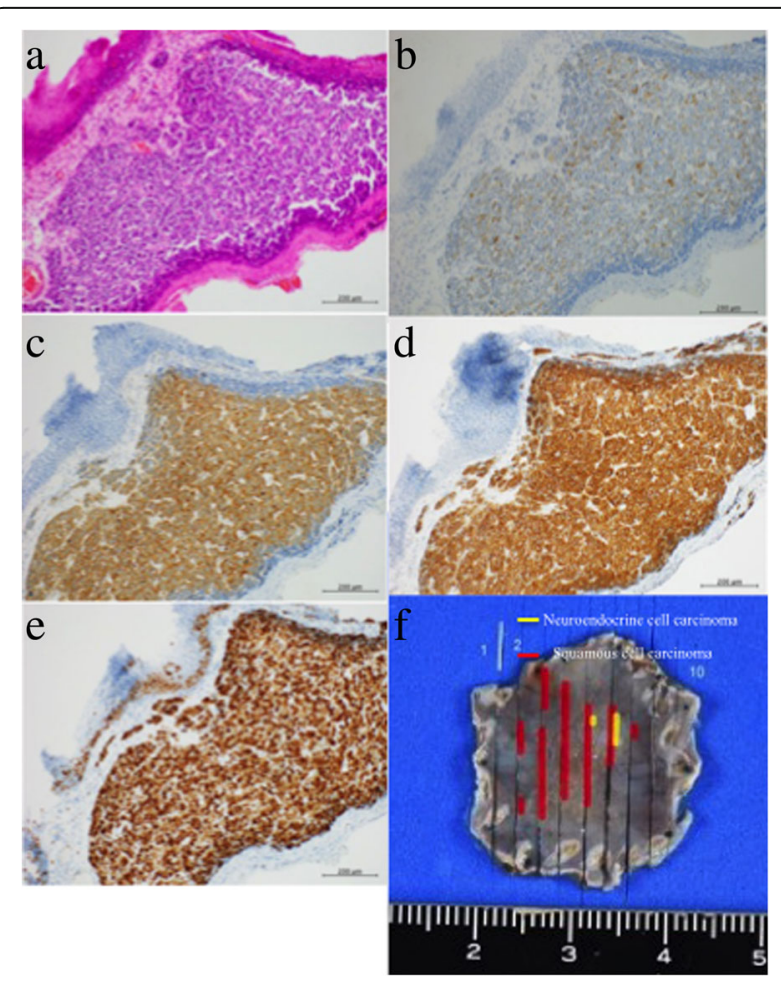

Fig. 2 Resected specimen by endoscopic submucosal dissection. This specimen shows neuroendocrine cell carcinoma arranged in a sheet fashion with mixed squamous cell carcinoma. Neuroendocrine cell carcinoma formed a duct and it is surrounded by squamous cell carcinoma. a Hematoxylin and eosin staining. Immunohistochemical staining showing: $\mathbf{b}$ chromogranin A, c synaptophysin, $\mathbf{d}$ CD56, and e Ki-67. $\mathbf{f}$ The fixed resected specimen is mapped by yellow and red lines. Red lines squamous cell carcinoma, yellow lines neuroendocrine cell carcinoma 
dissection from the cervix, mediastinum, and abdomen, and gastric conduit construction. Regional lymph node metastasis was identified in 1 of 76 nodes (number 108 lymph node), and the node metastasis stage was pN1. This lymph node contained NEC and no metastasis of SCC (Fig. 3a-d). After surgical treatment, he received chemotherapy consisting of etoposide and carboplatin for 3 months. He is alive and shows no sign of disease recurrence 12 months after surgery.

\section{Discussion}

NEC was formerly called small cell carcinoma. The first description of small cell carcinoma of the esophagus was reported in 1952 by McKeown [7]. The World Health Organization (WHO) definition for NEC includes positive endocrine markers, such as CD56, chromogranin A, and synaptophysin. A Ki-67 or mitotic index of $20 \%$ or higher is also necessary for diagnosing NEC. Tumors with less than $20 \% \mathrm{Ki}-67$ positivity are diagnosed as a neuroendocrine tumor [8].

NEC is categorized into two morphological types of small cell type and large cell type, and coexistence of SCC and/or adenocarcinoma is also often observed [9]. The small cell type is more aggressive than the large cell type, and it is frequently found in the advanced stage with lymph node and distant metastases [10].

We ascertained two clinically important issues based on the findings in our case. With regard to the first clinical issue, a small neuroendocrine tumor may occur with lymph node metastasis at an early stage, and attention should be paid to metastasis. Neuroendocrine tumors of the esophagus more frequently present in the middle of the esophagus and stain positive for CD56, synaptophysin, and chromogranin A $[1,11]$. These tumors are slow growing, but high-grade neuroendocrine tumors of the esophagus have a poor prognosis and a 5-year survival of approximately $25 \%[1,11]$. The therapeutic strategy for NEC of the esophagus has not been well defined because of the small number of cases [10]. To date, there has only been one case of collision of SCC and NEC of the esophagus treated by ESD [4].

Saddoughi et al. performed a retrospective analysis of malignant esophageal cancers including NEC [12]. They found that 1-year survival after surgical resection for rare types of malignant esophageal cancers was $69 \%$, 5 -year survival was $43 \%$, and 10 -year survival was $37 \%$. Surgical resection for rare types of esophageal malignancies should be considered part of effective treatment. In our case, a resected lymph node contained NEC without an SCC component, and regional lymph node metastasis was identified at the early stage of the NEC. Which patients will benefit from additional therapy at the early stage of NEC has not been well established. Therefore, additional therapy, such as esophagectomy and chemoradiotherapy, is required if histology from endoscopic resection shows a tumor invasion depth into the muscularis mucosa with lymphovascular invasion by NEC components.

Concerning the second clinical issue, the diagnosis of NEC before treatment is difficult. Table 1 summarizes cases of esophageal endocrine cell carcinoma treated by endoscopic resection. Characteristic endoscopic features

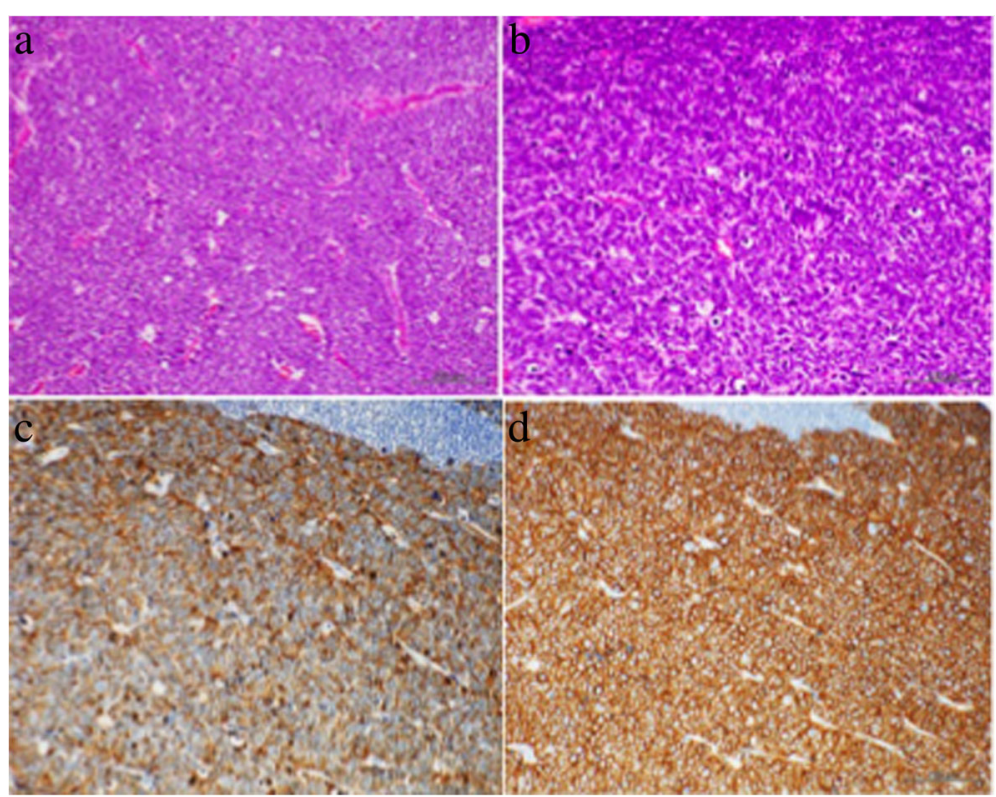

Fig. 3 Histological examination of a resected lymph node. a Low-power view and $\mathbf{b}$ high-power view (hematoxylin and eosin staining). Immunohistochemical staining shows: b chromogranin A, c synaptophysin, and d CD56 


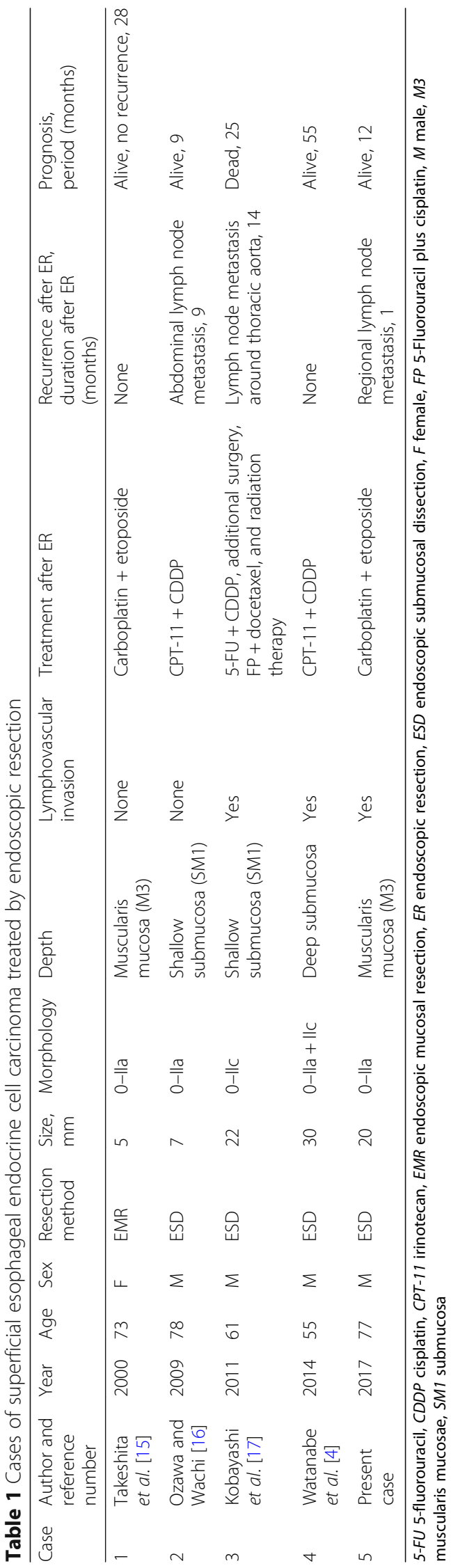


of NEC include submucosal growth, which is usually covered by normal epithelium with or without an ulcerous lesion in the center [8]. Biopsy sometimes fails to reach any diagnosis of NEC before treatment [4]. In our case, the microvascular pattern showed irregular, fine, reticular blood vessels near the center of the lesion. Vessels with a reticular pattern are defined as plexiform microvessels [13]. This vascular pattern is often found in invasive SCC or a non-SCC type of malignant epithelial neoplasm (for example, basaloid carcinoma, adenosquamous carcinoma, and endocrine tumor) with an infiltrative growth pattern [13, 14]. Therefore, vessels with a reticular pattern are not a specific sign for diagnosis of an early stage of NEC, but physicians have to consider the possibility of non-SCC types of malignancies.

\section{Conclusions}

In summary, we report a rare case of small superficial NEC showing regional lymph node metastasis. This case report highlights the fact that even if a NEC is small and limited to superficial, the tumor has the potential for metastasis if lymphovascular invasion by the NEC component occurs. In addition, this case indicates the necessity of close follow-up of small NEC after treatment.

\section{Abbreviations \\ CT: Computed tomography; EMR: Endoscopic mucosal resection; EP: Cancer limited to the epithelium; ESD: Endoscopic submucosal dissection; JES: Japan Esophageal Society; LPM: Cancer invading into the lamina propria; MM: Cancer invading into the muscularis mucosa; NBI: Narrow band imaging; NEC: Neuroendocrine cell carcinoma; SCC: Squamous cell carcinoma; WHO: World Health Organization}

\section{Acknowledgements}

We thank Nobuya Ohara for skillful technical assistance.

\section{Availability of data and materials}

Data sharing is not applicable to this article, because no datasets were generated or analyzed during the present study.

\section{Authors' contributions \\ SF was the major contributor to writing the manuscript. Endoscopic treatment was performed by MN and TY. The surgical treatment was performed by MK and $A M$. AY, AD, and HK supported the entire scope of work. TM reviewed and approved the final version of the manuscript. All authors read and approved the final manuscript.}

\section{Ethics approval and consent to participate}

Not applicable.

\section{Consent for publication}

Written informed consent was obtained from the patient for publication of this case report and any accompanying images. A copy of the written consent is available for review by the Editor-in-Chief of this journal.

\section{Competing interests}

The authors declare that they have no competing interests.

\section{Author details}

'Department of Gastroenterology, Kagawa Rosai Hospital, 3-3-1, Joutou, Marugame, Kagawa, Japan. ²Department of Surgery, Kagawa Rosai Hospital, Marugame, Kagawa, Japan. ${ }^{3}$ Department of Gastroenterology and Neurology، Faculty of Medicine, Kagawa University, Miki, Kagawa, Japan.

Received: 7 March 2018 Accepted: 18 July 2018

Published online: 24 August 2018

\section{References}

1. Huang Q, Wu H, Nie L, et al. Primary high-grade neuroendocrine carcinoma of the esophagus: a clinicopathologic and immunohistochemical study of 42 resection cases. Am J Surg Pathol. 2013;37:467-83.

2. Kukar M, Groman A, Malhotra U, et al. Small cell carcinoma of the esophagus: a SEER database analysis. Ann Surg Oncol. 2013;20:4239-44.

3. Yun JP, Zhang MF, Hou JH, et al. Primary small cell carcinoma of the esophagus: clinicopathological and immunohistochemical features of 21 cases. BMC Cancer. 2007;7:38

4. Watanabe K, Hikichi T, Sato M, et al. A case of endocrine cell carcinoma combined with squamous cell carcinoma of the esophagus resected by endoscopic submucosal dissection. Fukushima J Med Sci. 2014;60:187-91.

5. Inoue H, Sato $Y$, Sugaya $S$, et al. Endoscopic mucosal resection for early-stage gastrointestinal cancers. Best Pract Res Clin Gastroenterol. 2005;1 9:871-87.

6. Guo HM, Zhang XQ, Chen M, et al. Endoscopic submucosal dissection vs endoscopic mucosal resection for superficial esophageal cancer. World J Gastroenterol. 2014:20:5540-7.

7. Mckeown F. Oat-cell carcinoma of the oesophagus. J Pathol Bacteriol. 1952; 64:889-91.

8. Rindi G, Arnold R, Bosman FT, Capella C, Kilmstra DS, Klöppel G, Komminoth $P$, Solcia E. Nomenclature and classification of neuroendocrine neoplasms of the digestive system. In: Bosman FT, Carneiro F, Hruban RH, Theise ND, editors. WHO Classification of Tumours of the Digestive System. 4th ed. Lyon: IARC Press; 2010. p. 13-4.

9. Egashira A, Morita M, Kumagai R, et al. Neuroendocrine carcinoma of the esophagus: clinicopathological and immunohistochemical features of 14 cases. PLoS One. 2017;12:e0173501.

10. Brenner B, Tang LH, Klimstra DS, Kelsen DP. Small-cell carcinomas of the gastrointestinal tract: a review. J Clin Oncol. 2004;22:2730-9.

11. Lee CG, Lim YJ, Park SJ, et al. The clinical features and treatment modality of esophageal neuroendocrine tumors: a multicenter study in Korea. BMC Cancer. 2014;14:569.

12. Saddoughi SA, Taswell J, Harmsen WS, et al. Surgical resection of rare esophageal cancers. Ann Thorac Surg. 2016;101:311-5.

13. Arima M, Tada M, Arima H. Evaluation of microvascular patterns of superficial esophageal cancers by magnifying endoscopy. Esophagus. 2005; 2:191-7.

14. Oyama $T$, Inoue $H$, Arima $M$, et al. Prediction of the invasion depth of superficial squamous cell carcinoma based on microvessel morphology: magnifying endoscopic classification of the Japan Esophageal Society. Esophagus. 2017:14:105-12.

15. Takeshita S, Akahosi R, Sagara K, Oda T, Kiyozumi T, Kurano R, Mera K. A case of small cell carcinoma of the esophagus treated by endoscopic mucosal resection followed by chemotherapy (in Japanese with English abstract). Gastroenterol Endosc. 2000;42:258-61.

16. Ozawa T, Wachi E. Endocrine cell carcinoma of the esophagus resected by ESD after magnifying endoscopy with narrow band imaging (in Japanese with English abstract). Gastroenterol Endosc. 2009;51:2437-46.

17. Kobayashi K, Aoki T, Nishioka K, Takachi K, Ko-mori T, Hatano H, Yoshida K, Hayashi E. A case of esophageal endocrine cell carcinoma that recurred the lymph nodes around the thoracic aorta after ESD (in Japanese with English abstract). Gastroenterol Endosc. 2011;53:28-34.

\section{Publisher's Note}

Springer Nature remains neutral with regard to jurisdictional claims in published maps and institutional affiliations. 